\title{
Thickness distribution of the glenohumeral joint cartilage: a quantitative study using computed tomography
}

\author{
Valentin Zumstein • Marko Kraljević • \\ Annemarie Conzen · Sebastian Hoechel · \\ Magdalena Müller-Gerbl
}

Received: 26 February 2013/ Accepted: 14 October 2013/Published online: 31 October 2013

(C) Springer-Verlag France 2013

\begin{abstract}
Purpose Among late signs like sclerosis, cysts and osteophytes, alteration of cartilage is a common problem in osteoarthritis. To detect abnormal states in the glenohumeral joint, the physiologic distribution of the cartilage thickness must be known, which will allow physicians to better advise patients. High-resolution computed tomography (CT) data in soft tissue kernel provide highly accurate quantitative results and are a useful method to determine the geometrical situation of the glenohumeral joint. The objective of this study was to characterize the distribution of the thickness of the glenohumeral joint cartilage using CT.

Methods To investigate the distribution of thickness of the joint cartilage, CT images in soft tissue kernel of nine specimens were analyzed using image visualization software. Statistical analysis of the obtained data was performed using the ANOVA test.

Results Results showed different patterns in the glenoid cavity than in humeral head. Cartilage thickness in all glenoids showed maxima in the inferior and anterior portion, whereas central areas are covered with the thinnest cartilage layer. Maximum cartilage thickness in the humeral head was found in the central and superior parts.

Conclusion We could show that the distribution of cartilage thickness in the glenohumeral joint is not
\end{abstract}

V. Zumstein and M. Kraljević contributed equally to this work.

V. Zumstein · M. Kraljević $(\bowtie) \cdot$ S. Hoechel · M. Müller-Gerbl Institute of Anatomy, University of Basel, Pestalozzistrasse 20, 4056 Basel, Switzerland

e-mail: marko.kraljevic@gmail.com

\section{A. Conzen}

Institute of Anatomy, Ludwig Maximilian University Munich, Pettenkoferstrasse 11, 80336 Munich, Germany homogenous and that there exist several reproducible patterns. Evaluation of cartilage thickness in the glenohumeral joint is of high interest in basic and clinical research.

Keywords Cartilage thickness - Glenohumeral joint . Osteoarthritis · Computed tomography

\section{Introduction}

Among late signs like sclerosis, cysts and osteophytes, alteration of cartilage is a common problem in osteoarthritis. It could be shown that a change in loading after rotator cuff injury leads to changes in cartilage thickness and subsequently to severe damage of the joint [15]. Detection of cartilage thickness is of high interest in basic and clinical research. Quantitative cartilage thickness measurement is important to improve the outcome of shoulder arthroplasty, to evaluate functional adaption to altered mechanical loading or to detect individuals with high risk of cartilage wear [6].

Several authors showed that under decreased loading conditions, the hyaline cartilage undergoes a process of thinning, whereas increased loading situations prevent cartilage from degeneration, although they will not lead to a thickened cartilage $[5,16]$. Investigations comparing a group of paraplegics to tetraplegics confirmed those findings. The mean humeral cartilage thickness in individuals with paraplegia was significantly greater in the superior area compared to individuals with quadriplegia, where the inferior areas showed relatively greatest cartilage thickness [16]. These findings could be explained by the fact that superior cartilage portion becomes thinner due to a lack of loading maxima in this region. 
Table 1 Clinical data of all specimens

\begin{tabular}{llll}
\hline Specimen & Age & Sex & Side \\
\hline 1 & 31 & M & Right \\
2 & 63 & M & Right \\
3 & 33 & F & Right \\
4 & 62 & M & Right \\
5 & 45 & M & Right \\
6 & 20 & M & Right \\
7 & 45 & F & Right \\
8 & 45 & M & Right \\
9 & 24 & M & Right \\
\hline
\end{tabular}

In the past, stereophotogrammetry (SPG) technique was used to provide information about the articular geometry of the glenohumeral joint [18]. Other authors described cartilage situations in human joints using three-dimensional (3D) gradient echo MRI sequences with selective water excitation $[6,16]$. In this study, we provide highly accurate quantitative results using high-resolution computed tomography (CT) data in soft tissue kernel. This method allows determining the distribution of articular cartilage thickness as well as evaluation of the subchondral bone plate [12].

Our objective was to characterize the distribution of the glenohumeral cartilage thickness using CT. Based on the findings of Soslowsky et al. [18] and Graichen et al. [6], we hypothesized that $(\mathrm{H} 1)$ the cartilage thickness of the glenoid cavity has its maximal values in the peripheral areas and $(\mathrm{H} 2)$ the humeral head will be thickest in the central part.

\section{Materials and methods}

\section{Specimens analyzed}

This study included nine fresh shoulder specimens (age 20-63 years, mean age 41 years, 2 females and 7 males) from the Institute of Forensic Medicine of Ludwig Maximilian University in Munich (Table 1). The interval between death and investigation was kept to $48 \mathrm{~h}$ at most. All investigated shoulder joints were from the right side and were previously checked by observation to exclude specimens showing obvious signs of degeneration or signs of joint instability (Hill-Sachs or Bankart lesion).

Cartilage thickness measurement

The specimens were scanned axially in a CT scanner (Siemens Somatom Plus 4; slice thickness $2.0 \mathrm{~mm}$; peak $120 \mathrm{kV}$; X-ray tube current $130 \mathrm{~mA}$; convolution kernel 59). The obtained data sets were transferred into an image analyzing system. We imported the ACR NEMA images in soft tissue kernel to on-display measurement using the image visualization software VGStudio Max 2.1.1. (Volume Graphics GmbH, Heidelberg, Germany). Surface determination and orientation of the specimens were performed using the method described by Nowakowski et al. [13] and De Wilde et al. [4] (Fig. 1a).

A standardized grid $(5 \times 7$ units $)$ was projected onto the joint surface. At each measuring point (Fig. 1b), a line perpendicular to the joint surface was fitted to determine cartilage thickness (Fig. 1c). This allowed the evaluation of the cartilage thickness distribution in detail and to compare the results in summary charts.

\section{Statistical analysis}

In a first step, the values of maximum thickness of cartilage of each joint were visually compared with the other specimens in a summary chart $(5 \times 7$ units $)$ (Fig. 2). In addition, mean values and standard deviations of all specimens were calculated for each measuring point, and for better visualization, a diagram including all information was built (Fig. 3). In this diagram, false colors were assigned to the
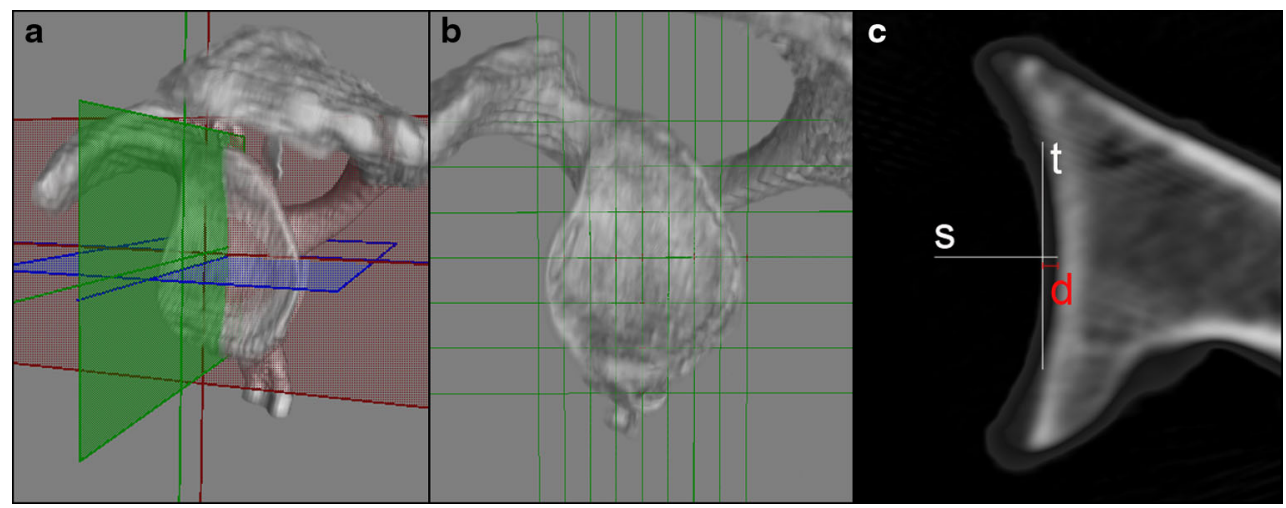

Fig. 1 Procedure of the image visualization software VGStudio Max: a alignment of the anatomical planes in relation to the articular surface, b projection of a standardized grid onto the joint surface, c measurement $(d)$ of the cartilage thickness using a tangent to the cartilage surface $(t)$ and a perpendicular line $(s)$ to the articular surface 
measured cartilage thickness values at each measuring point. Statistical analysis of these data was performed using the ANOVA test. The level of significance was set at $p<0.05$.
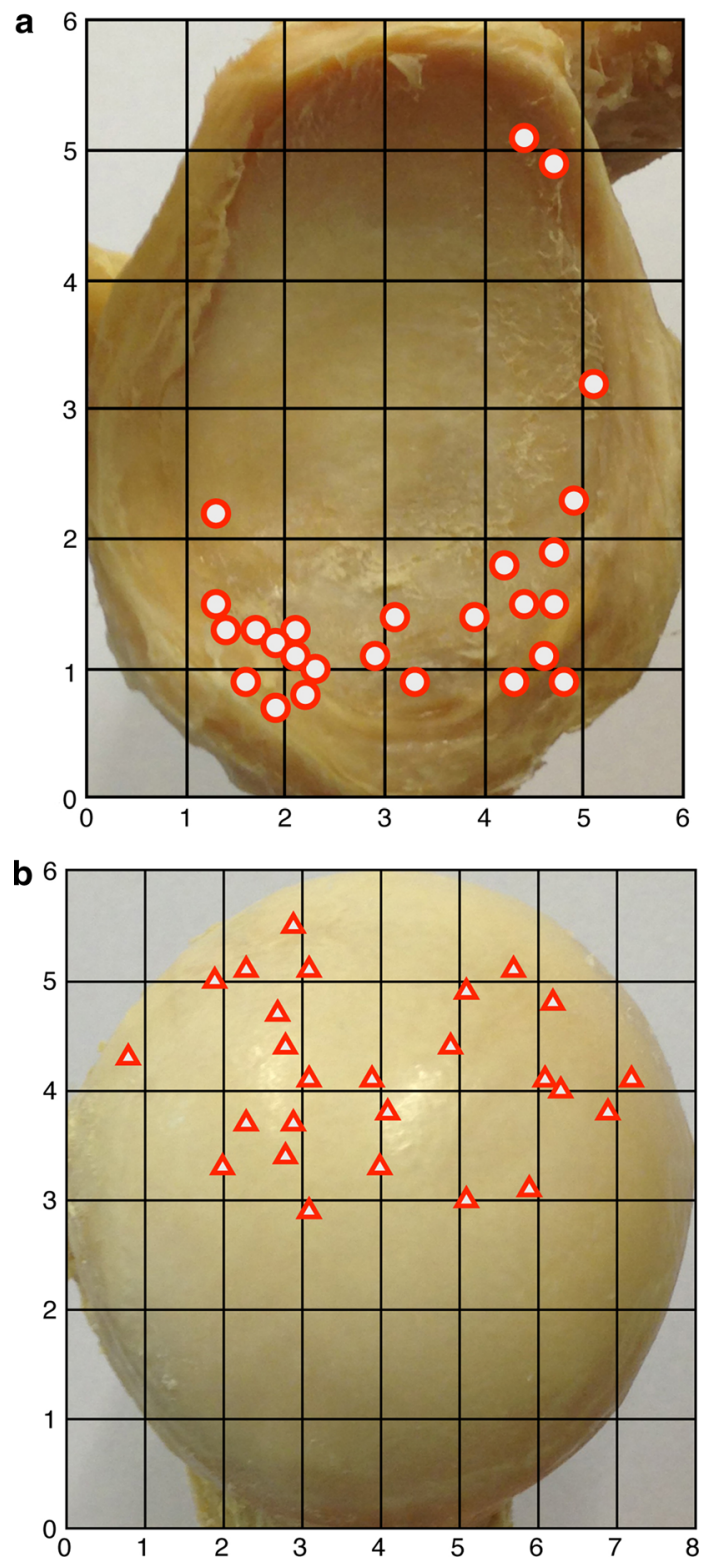

Fig. 2 Summary chart of all cartilage thickness maxima in a glenoid cavities and $\mathbf{b}$ humeral heads

\section{Results}

The mean cartilage thickness revealed values of $1.93 \mathrm{~mm}$ (SD $0.59 \mathrm{~mm}$ ) for the glenoid cavity and $1.74 \mathrm{~mm}$ (SD $0.45 \mathrm{~mm}$ ) for the humeral head. The evaluation of cartilage distribution revealed areas of high and low thickness. In nine glenoid cavities, the cartilage thickness was highest in the inferior and anterior parts. The mean values for the cartilage thickness were $2.54 \mathrm{~mm}(\mathrm{SD} 0.50 \mathrm{~mm})$ for the inferior area, $2.10 \mathrm{~mm}$ (SD $0.90 \mathrm{~mm}$ ) for the superior area, $1.82 \mathrm{~mm}(\mathrm{SD} 0.51 \mathrm{~mm})$ for the posterior area, $2.30 \mathrm{~mm}$ (SD $0.66 \mathrm{~mm}$ ) for the anterior area and $1.61 \mathrm{~mm}$ (SD $0.40 \mathrm{~mm}$ ) for the central area. The CT-based assessment of the cartilage thickness in the glenoid cavity varied from $0.98 \mathrm{~mm}$ at its thinnest point to $4.80 \mathrm{~mm}$ at its thickest measuring point (Fig. 3a).

The localization of the thickest cartilage parts in nine humeral heads was found to be superior and central. The mean values for the humeral head were $1.44 \mathrm{~mm}$ (SD $0.38 \mathrm{~mm}$ ) for the inferior area, $1.96 \mathrm{~mm}$ (SD $0.68 \mathrm{~mm}$ ) for the superior area, $1.73 \mathrm{~mm}(\mathrm{SD} 0.41 \mathrm{~mm})$ for the posterior area, $1.89 \mathrm{~mm}(\mathrm{SD} 0.60 \mathrm{~mm})$ for the anterior area and $1.78 \mathrm{~mm}(\mathrm{SD} 0.43 \mathrm{~mm}$ ) for the central area. The cartilage thickness of the humeral head ranged from 0.92 to $3.32 \mathrm{~mm}$ (Fig. 3b).

We found no statistical significant age- or gender-related differences in absolute cartilage thickness and distribution patterns.

All differences showed statistical significance $(p<0.05)$.

\section{Discussion}

Alteration of cartilage is a common problem in osteoarthritis of the shoulder joint. Altered loading after rotator cuff injury leads to transformations in cartilage thickness and to severe damage of the joint [15]. Hyaline cartilage requires close regulation of both architecture and biomechanical composition [14]. It is well known that moderate loading of joints helps maintain cartilage whereas excessive chronic mechanical loading is thought to be a risk
Fig. 3 Distribution of cartilage thickness (in $\mathrm{mm}$ ) in all a glenoids and $\mathbf{b}$ humeral heads (summary picture) a

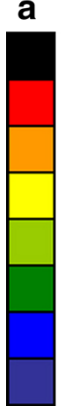

$>2.84$

$2.52-2.84$

$2.20-2.52$

$1.88-2.20$

$1.56-1.88$

$1.24-1.56$

$0.92-1.24$

$<0.92$

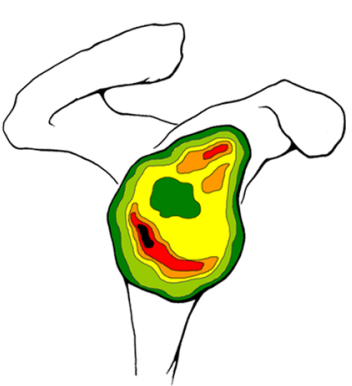

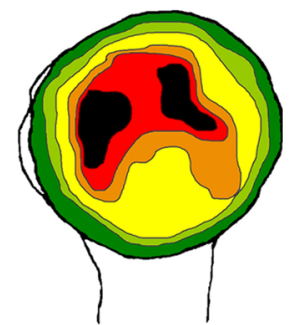


factor for the onset and progression of osteoarthritis [19]. Detection of cartilage thickness is of high interest in basic and clinical research. Quantitative cartilage thickness measurement is important for the evaluation of functional adaption to altered mechanical loading or detection of individuals with a high risk of cartilage wear and improvement of shoulder arthroplasty outcome [6].

In this study, we used a soft tissue kernel-based highresolution $\mathrm{CT}$ and postprocessing technique. We found a high level of agreement regarding maximal cartilage thickness as well as cartilage distribution patterns compared to studies using MRI, SPG [2, 18], A-mode ultrasound [1, 9, 11, 17] or on anatomical sections [7]-based cartilage measurement. A-mode ultrasound has been shown to be very accurate, particularly due to its high spatial resolution $[1,11,17]$. Studies with qMRI on cadaveric shoulder specimens revealed lower values of mean cartilage thickness compared to A-mode investigations [6]. When comparing the values for mean and maximum cartilage thickness in the shoulder using SPG [18] to the qMRI results, the results of qMRI were lower as well [6]. Our results confirmed these findings. The mean and maximum cartilage thickness revealed somewhat higher values compared to the findings in qMRI (humerus: mean, 1.2 vs. $1.7 \mathrm{~mm}$; maximum, 2.3 vs. $3.3 \mathrm{~mm}$ in our study and glenoid cavity: mean, 1.7 vs. $1.9 \mathrm{~mm}$; maximum, 3.1 vs. $4.8 \mathrm{~mm}$ ).

In our study, maximal cartilage thickness of the humeral head was localized in the superior and central areas of the joint surface. Studies of Ruckstuhl et al. [16] confirmed those findings. The mean humeral cartilage thickness in individuals with paraplegia was significantly greater in the superior area compared to individuals with quadriplegia, where the inferior areas showed relatively greatest cartilage thickness [16]. These findings could be explained by the fact that the superior cartilage portion becomes thinner due to the lack of loading maxima in this region. Several authors showed that the hyaline cartilage undergoes a process of thinning under decreased loading conditions, whereas increased loading situations prevent cartilage from degeneration, although they will not lead to a thickened cartilage [5].

In the glenoid cavity, maximum cartilage thickness values were recorded in the periphery of the joint surface, particularly in the inferior and anterior parts. Investigations of different authors $[6,18]$ support our findings. Soslowsky et al. [18] described that measurements taken from radiographs pretend a more flat glenoid compared to the corresponding humeral head, but cartilage thickness measurements can explain the observed incongruity of humeral head and glenoid cavity seen on radiographs. Recent studies investigating the mineralization patterns in the subchondral bone plate of the glenohumeral joint as a marker of long-term stress distribution $[8,20]$ reported recurring patterns with anterior and posterior mineralization maxima, which might be explained by a moderate anterior-posterior shifting of the humeral head during range of motion. A combination of this information with the method described in the work of Billuart et al. [3] could provide interesting information about the glenohumeral biomechanics and conformity during different ranges of motion.

We could not find any significant age- or gender-related differences in cartilage thickness or distribution. Further studies with a larger amount of investigated specimens will show whether previously described gender differences in glenoid anatomy are also applicable to cartilage thickness distribution [10].

In conclusion, this work presents a method to provide precise and valuable information about cartilage thickness distribution in human joints. Our obtained results for the glenohumeral joint are in agreement with those of several in vitro studies using qMRI or SPG. For screening, diagnosis and follow-up of osteoarthritis, cartilage thickness measurements are of great importance both in healthy and diseased shoulder joints.

Conflict of interest The authors declare that they have no financial and personal relationships with other people or organizations that could inappropriately influence their work.

\section{References}

1. Adam C, Eckstein F, Milz S, Putz R (1998) The distribution of cartilage thickness within the joints of the lower limb of elderly individuals. J Anat 193(Pt 2):203-214

2. Ateshian GA, Soslowsky LJ, Mow VC (1991) Quantitation of articular surface topography and cartilage thickness in knee joints using stereophotogrammetry. J Biomech 24(8):761-776

3. Billuart F, Devun L, Gagey O, Skalli W, Mitton D (2007) 3D kinematics of the glenohumeral joint during abduction motion: an ex vivo study. Surg Radiol Anat 29(4):291-295. doi:10.1007/ s00276-007-0208-2

4. De Wilde L, Defoort S, Verstraeten TR, Speeckaert W, Debeer P (2012) A 3D-CT scan study of the humeral and glenoid planes in 150 normal shoulders. Surg Radiol Anat 34(8):743-750. doi:10. 1007/s00276-011-0836-4

5. Eckstein F, Hudelmaier M, Putz R (2006) The effects of exercise on human articular cartilage. J Anat 208(4):491-512. doi:10. 1111/j.1469-7580.2006.00546.x

6. Graichen H, Jakob J, von Eisenhart-Rothe R, Englmeier KH, Reiser M, Eckstein F (2003) Validation of cartilage volume and thickness measurements in the human shoulder with quantitative magnetic resonance imaging. Osteoarthr Cartil 11(7):475-482

7. Hodler J, Loredo RA, Longo C, Trudell D, Yu JS, Resnick D (1995) Assessment of articular cartilage thickness of the humeral head: MR-anatomic correlation in cadavers. AJR Am J Roentgenol 165(3):615-620. doi:10.2214/ajr.165.3.7645480

8. Kraljevic M, Zumstein V, Hugli R, Muller-Gerbl M (2012) A comparison of subchondral bone mineralization between the glenoid cavity and the humeral head on 57 cadaverous shoulder joints. Surg Radiol Anat. doi:10.1007/s00276-012-1034-8 
9. Mann RW (2001) Comment on 'ultrasonic measurement of the thickness of human articular cartilage in situ' by Yao and Seedhom. Rheumatology (Oxford) 40(7):829-831

10. Merrill A, Guzman K, Miller SL (2009) Gender differences in glenoid anatomy: an anatomic study. Surg Radiol Anat 31(3):183-189. doi:10.1007/s00276-008-0425-3

11. Modest VE, Murphy MC, Mann RW (1989) Optical verification of a technique for in situ ultrasonic measurement of articular cartilage thickness. J Biomech 22(2):171-176

12. Muller-Gerbl M, Putz R, Hodapp N, Schulte E, Wimmer B (1989) Computed tomography-osteoabsorptiometry for assessing the density distribution of subchondral bone as a measure of longterm mechanical adaptation in individual joints. Skeletal Radiol 18(7):507-512. doi:10.1007/BF00351749

13. Nowakowski AM, Muller-Gerbl M, Valderrabano V (2012) Assessment of knee implant alignment using coordinate measurement on three-dimensional computed tomography reconstructions. Surg Innov 19(4):375-384. doi:10.1177/1553350611 429689

14. Pollard TC, Gwilym SE, Carr AJ (2008) The assessment of early osteoarthritis. J Bone Joint Surg Br 90(4):411-421. doi:10.1302/ 0301-620X.90B4.20284
15. Reuther KE, Sarver JJ, Schultz SM, Lee CS, Sehgal CM, Glaser DL, Soslowsky LJ (2012) Glenoid cartilage mechanical properties decrease after rotator cuff tears in a rat model. J Orthop Res 30(9):1435-1439. doi:10.1002/jor.22100

16. Ruckstuhl H, Krzycki J, Petrou N, Vanwanseele B, Stussi E (2008) A quantitative study of humeral cartilage in individuals with spinal cord injury. Spinal Cord 46(2):129-134. doi:10.1038/ sj.sc. 3102084

17. Rushfeldt PD, Mann RW, Harris WH (1981) Improved techniques for measuring in vitro the geometry and pressure distribution in the human acetabulum-I. Ultrasonic measurement of acetabular surfaces, sphericity and cartilage thickness. J Biomech 14(4):253-260

18. Soslowsky LJ, Flatow EL, Bigliani LU, Mow VC (1992) Articular geometry of the glenohumeral joint. Clin Orthop Relat Res 285:181-190

19. Sun HB (2010) Mechanical loading, cartilage degradation, and arthritis. Ann NY Acad Sci 1211:37-50. doi:10.1111/j.17496632.2010.05808.x

20. Zumstein V, Kraljevic M, Huegli R, Muller-Gerbl M (2011) Mineralisation patterns in the subchondral bone plate of the humeral head. Surg Radiol Anat 33(9):775-779. doi:10.1007/ s00276-011-0819-5 